\title{
New limits on coloured three jet resonances
}

\author{
Hassan Easa, Thomas Gregoire and Daniel Stolarski \\ Ottawa-Carleton, Institute for Physics, Carleton University, \\ 1125 Colonel By Drive, Ottawa, ON, K1S 5B6, Canada \\ E-mail: Hassaneasa@cmail.carleton.ca, gregoire@physics.carleton.ca, \\ stolar@physics.carleton.ca
}

\begin{abstract}
We consider experimental limits on colour triplet fermions that decay dominantly to three jets via a scalar mediator that can be on- or off-shell. These fermions arise in top-partner models that can solve the hierarchy problem, and limits on this scenario are weaker than those on traditional top-partner models because of the messy all-hadronic final state with significant backgrounds. We do find, however, that while there are no dedicated searches for this scenario, especially in case of an on-shell mediator, the suite of LHC allhadronic searches still constrains a significant portion of the parameter space. In particular, we find that searches for pair production of di-jet and tri-jet resonances are complementary, covering different regions of parameter space. We also find that if the final state is rich in $b$ jets, current limits do not change significantly relative to the scenario with all light jets, and we describe how modifications of current search strategies can improve limits in that case.
\end{abstract}

KeYwords: Jets, Phenomenological Models

ARXIV EPRINT: 2003.00014 


\section{Contents}

1 Introduction $\quad 1$

2 Bounds from LHC searches 3

2.1 CMS pair-produced three-jet resonances 4

2.2 ATLAS pair-produced di-jet resonances 8

3 Heavy flavour scenario $r$

4 Conclusion $\quad 12$

$\begin{array}{ll}\text { A CMS three jet resonance search } & 13\end{array}$

B ATLAS di-jet resonance search $\quad 15$

C CMS three jet resonance search at $\sqrt{s}=8 \mathrm{TeV} \quad 16$

$\begin{array}{ll}\text { D Multijet background } & 17\end{array}$

\section{Introduction}

With the discovery of the Higgs boson [1,2] at the large hadron collider (LHC), the Standard Model of particle physics (SM) is complete. The confirmation of the properties of the Higgs being SM-like and the lack of discovery of new physics at the TeV scale exacerbates the hierarchy problem: what cuts off the quantum corrections to the Higgs mass? One well known solution to the hierarchy problem poses the existence of fermionic top partners, fermions with the same quantum numbers as the top quark whose contributions to the Higgs mass parameter cancel those of the top quark. These can appear in composite Higgs models [3-7] and Little Higgs models [8, 9].

In these models, top partners typically decay to a top quark and a Higgs or $Z$, or to a bottom quark and $W$. LHC searches for top partners in these modes are extensive, both in pair production [10-19] and in single production [20-30], with limits $\approx 1.3-1.66 \mathrm{TeV}$ on the mass of the top partners from the various searches depending on their branching ratios. Due to the lack of discovery, it is critical to explore alternative models, particularly those with different decay modes for the top partners. One could imagine, for example, decays involving a charged or neutral scalar [31-33], decays involving a top quark in association with a gluon or a photon $[34,35]$, a neutral boson that subsequently decays to two photons [36], a dark photon or a dark Higgs [37], or a pseudo-scalar which promptly decays to a pair of gluons or b-quarks [38-40]. In this work we consider the particularly challenging 


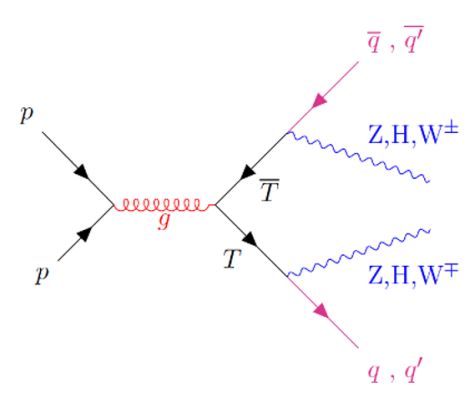

(a) Conventional decay modes.

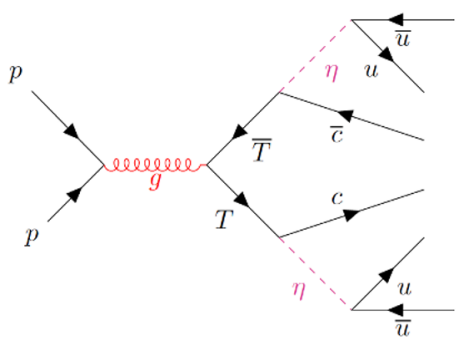

(b) Our model.

Figure 1. Feynman diagrams for the conventional decay modes and the processes considered in this letter.

possibility of a final state with only hadronic activity and no leptons or missing energy and study the limits on the masses of such top partners.

We consider a simplified model for our top partners $(T)$ which contains the following processes: $T$ is pair produced via the strong interactions, $p p \rightarrow T \bar{T}$. It then decays to a light flavour quark $(j)$ and a new scalar $\eta$, and that scalar decays to two light flavour jets. The full process is

$$
p p \rightarrow T \bar{T} \rightarrow j j \eta \eta \rightarrow 6 j,
$$

with a representative Feynman diagram shown in the right panel of figure 1. In our studies, we consider the decay channel $T \rightarrow j j j$ where $j$ can be associated with any light quark. However, if one were to focus mainly on the decay channel $T \rightarrow c j j$, the limits obtained from considering charm tagging will not significantly improve the sensitivity of the signal or alter the limits obtained, mainly because charm tagging is notoriously difficult and the efficiency is significantly worse than $b$-tagging. For example, the $c$-tagging efficiency is approximately $20 \%$ for about $1 \%$ light jet misidentification rate, where as the $b$-tagging efficiency can be approximately $70 \%$ at about $1 \%$ misidentification rate [41-44]. Note the tagging efficiencies depends on jet $p_{T}$ and $\eta$. An explicit model which gives rise to this signature (without associated top and bottom signatures) and solves the hierarchy problem is given in [45]. This scenario can be thought of as the fermionic analogue of hadronic $R$-parity violation (RPV) [46-48] in Supersymmetry where the top squark can decay to two light jets.

This six-jet final state is experimentally very challenging as the QCD multijet background is very large and difficult to determine. While one might expect that the limits for this model are significantly weaker than for the traditional decay modes, we will show that these models are also strongly constrained, with the best limits coming from recasting searches for RPV gluino searches from CMS [49] and ATLAS [50]. The RPV gluino has the same signal topology as eq. (1.1), but the cross section for a colour octet is larger than for a colour triplet top partner. In this work we will study various qualitatively different regions of parameter space including:

- Off-shell $\eta: m_{\eta} \gg m_{T}$,

- Bulk on-shell region: $m_{\eta} \lesssim m_{T}$, 
- Very light $\eta: m_{\eta} \ll m_{T}$,

- Degenerate region: $m_{\eta} \sim m_{T}$.

We will show that all of these regions are constrained up to a $T$ mass of about 700 to $900 \mathrm{GeV}$.

We also consider the possibility that the scalar particle $(\eta)$ decays to two bottom jets instead of light jets, as might be expected from a Higgs-like scalar. The complete process for this particular decay mode is:

$$
p p \rightarrow T \bar{T} \rightarrow j j \eta \eta \rightarrow 2 j 4 b .
$$

From the presence of the two $b$-jets in the final state, one might expect that the corresponding limit on $T$ would be stronger than in the light jets case, but in fact the constraints are very similar. Adding $b$-tagging to current search strategies can significantly improve limits on this scenario.

This paper is structured as follows. In section 2 we present the bounds coming from the latest LHC searches for top partners decaying exclusively to light jets. In particular, we consider searches looking for pairs of resonances decaying to three jets, and searches looking for pairs of di-jet resonances. In section 3 we repeat this exercise for final state containing $b$-jets. In section 4 we give a brief summary of the results. This work is augmented by four appendices: in appendix A we give some details regarding the three-jet CMS resonance search performed at $\sqrt{s}=13 \mathrm{TeV}$, in appendix $\mathrm{B}$ we present all the selection requirements for the di-jet ATLAS search applied to our model, in appendix $\mathrm{C}$ we discuss the three-jets CMS search conducted at $\sqrt{s}=8 \mathrm{TeV}$, and in appendix $\mathrm{D}$ we give details on how QCD background events are simulated.

\section{Bounds from LHC searches}

The topologies we consider here consist of resonances that ultimately decay fully hadronically leading to a six-jet final state, not counting initial and final state radiation. Furthermore, we assume that the scalar $(\eta)$ decays promptly; hence, resulting in the absence of any displaced vertices. This multijet final state narrows down the list of possible searches sensitive to this model. The pertinent searches to consider are the ones looking for multiple jets but no missing energy, leptons, or photons.

In order to recast existing searches, we use a few publicly available software packages/tools. The model file for our model was created using the Mathematica package FeynRules [51] which was then supplied as an input to MadGraph5 [52] for Monte Carlo (MC) event generation. Next, the events were passed to PYTHIA 8 for showering and hadronization [53]; subsequently, DELPHES 3 [54] was used for fast detector simulation and FastJet [55] was deployed to reconstruct jets.

The top partner pair production cross section was computed at next-to-leading order (NLO) using MadGraph5 by setting the top quark mass to $m_{T}$ and its behaviour as a function of the top partner mass is shown in figure 2. The theoretical cross sections 


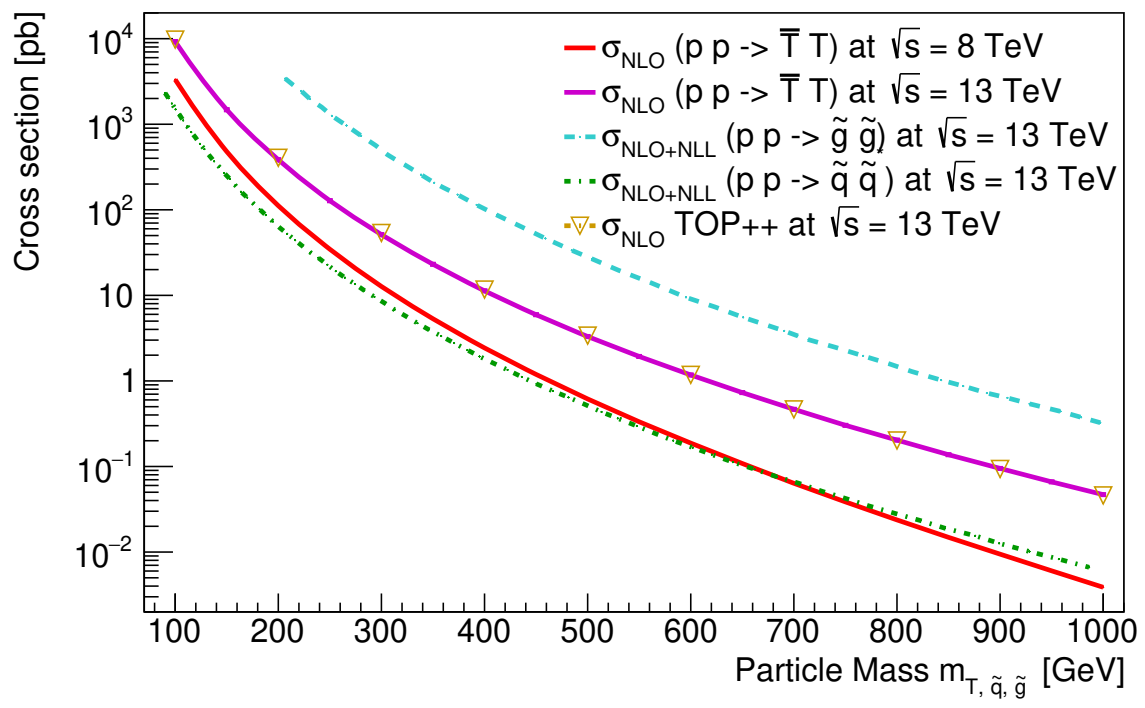

Figure 2. Next-to-leading order (NLO) pair production cross section for the top partner as a function of $m_{T}$ at $\sqrt{s}=8,13 \mathrm{TeV}$. The cross section was computed using MadGraph5 at next-toleading order by varying the top quark mass. The figure also contains the NLO top partner pair production cross section at $\sqrt{s}=13 \mathrm{TeV}$ computed using TOP ++2.0 program for a few benchmark points (triangles). Error bars from varying the renormalization and factorization scales are included but too small to see. The next-to-leading order plus next-to-leading-logarithm (NLL) cross sections for gluino [49] and squark [50] pair production at $\sqrt{s}=13 \mathrm{TeV}$ are also shown for comparison.

were also computed with the $\mathrm{TOP}++2.0$ program [56] for comparison and were found to be consistent. The benchmark points displayed in figure 2 contain statistical and scale uncertainties from renormalization and factorization scale $\left(\mu_{F}\right.$ and $\mu_{R}$ are varied from 0.5 to 2 times the nominal value). The error bars are too small to observe on the plot. Furthermore, the corresponding values obtained were confirmed by the available literature computations for top partner pair production cross sections [10, 14, 16, 17].

\subsection{CMS pair-produced three-jet resonances}

Searches that explore the fully hadronic decay channels with at least six jets (light) jets in the final state have been conducted at the LHC. Older searches include CMS [57] and ATLAS $[58,59]$ searches at $\sqrt{s}=7,8 \mathrm{TeV}$ that place no constraint on our signal. A similar conclusion was reached by the authors for the double trijet resonances for the composite models in [60] which considers the CMS search [57]. However, their topology is slightly different since it involves a color octet rather than a singlet $\eta$. The latest multijet search that matches our desired search criteria was conducted by CMS using $35.9 \mathrm{fb}^{-1}$ of data collected at a $13 \mathrm{TeV}$ center-of-mass energy [49]. The search is designed to look for a pair of particles each decaying to three jets. The analysis interprets the results in the framework of an R-parity violating (RPV) SUSY model where gluinos are pair produced and each decay to three quarks, resulting in a six-jet final state. The search explores a gluino mass range from 200 to $2000 \mathrm{GeV}$ and excludes gluino masses below $1500 \mathrm{GeV}$ at $95 \%$ confidence 


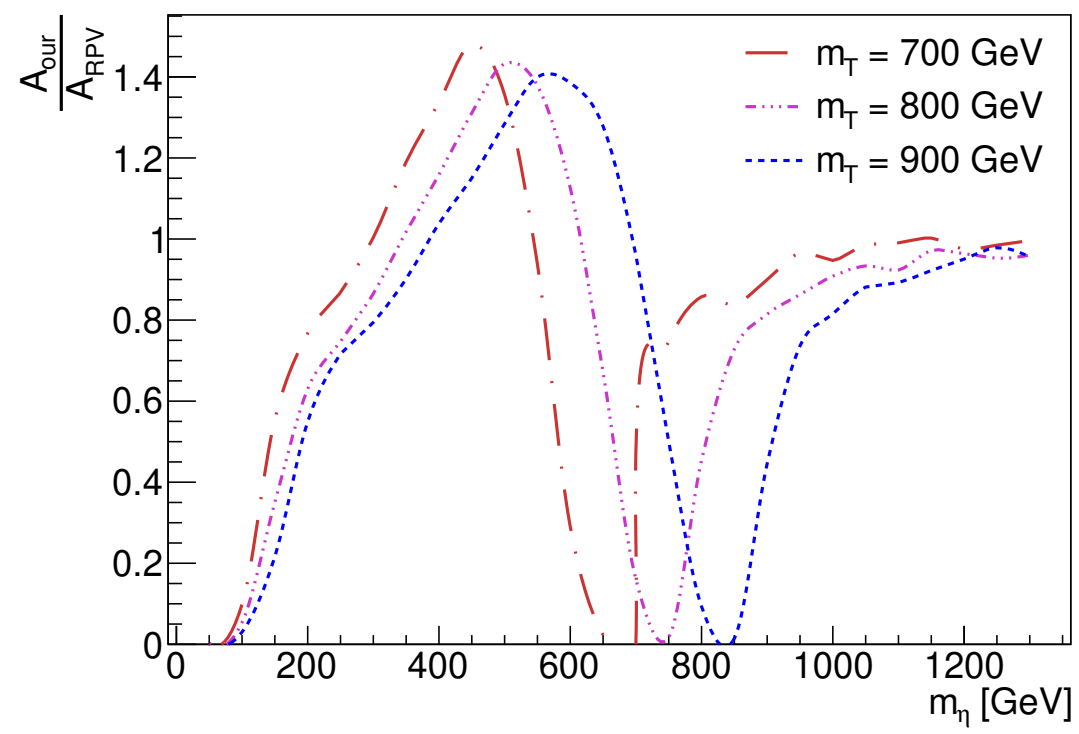

Figure 3. The ratio of acceptance in the recasted CMS search [49] for our model over the RPV benchmark model as a function of the scalar mass for a few top partner masses.

level. This dedicated analysis focuses on three-jet resonances and takes advantage of Dalitz variables [61] to enhance signal sensitivity. A distance parameter, sensitive to the symmetry of the jets inside a triplet is defined as:

$$
D_{[3,2]}^{2}=\sum_{i>j}\left(\hat{m}(3,2)_{i j}-\frac{1}{\sqrt{3}}\right)^{2},
$$

where $\hat{m}(3,2)_{i j}$ is the normalized di-jet invariant masses and is defined as:

$$
\hat{m}(3,2)_{i j}^{2}=\frac{m_{i j}^{2}}{m_{i}^{2}+m_{j}^{2}+m_{k}^{2}+m_{i j k}^{2}}, \quad \text { where } i, j, k \in\{1,2,3\} .
$$

Here, $m_{i j k}$ is the triplet invariant mass and $m_{i}$ are the constituent jet masses of the triplet. The complete list of selection criteria used in this particular search are given in table 2 in the appendix. To set bounds, the QCD and combinatorial backgrounds are modelled with a monotonically decreasing function, which is optimized in four mass regions. A statistically significant signal-like "bump", parametrized by a double Gaussian, is then looked for on top of this background.

In order to recast this search and obtain bounds on the parameter space of our model, we first simulated the RPV SUSY topology given in [49] with all superpartners except the gluino decoupled. We then simulated our particular model for the corresponding $m_{T}$ $\left(m_{T}=m_{\tilde{g}}\right)$ for a fixed $m_{\eta}$. We computed the acceptance, defined as the number of correct triplets passing all the selection criteria given in table 2 divided by the total number of events generated, for both the original RPV topology and our model. The correct triplets are the ones constructed from the three jets associated with the decay of a gluino (for 
the CMS topology) or a top partner (for our topology). They have an invariant mass distribution peaked around the resonance mass. ${ }^{1}$ In order to identify these correct triplets, we require the parton level decay products to be within $\Delta R=0.3$ from the detector level jet axis. We then rescale the pair production cross section for $\bar{T} T$ shown in figure 2 by the ratio of the acceptances as:

$$
\sigma_{\text {rescaled }}=\sigma(p p \rightarrow \bar{T} T) \times \frac{\mathcal{A}_{\text {our }}}{\mathcal{A}_{R P V}},
$$

where $\mathcal{A}_{\text {our }}$ is the acceptance for our topology and $\mathcal{A}_{R P V}$ is the acceptance obtained by simulating the RPV benchmark model in [49]. Because the invariant mass distribution of correct triplets for our topology, including cases where $\eta$ is on-shell, is very similar to the RPV topology, the number of events that our model would produce in an invariant mass peak distinguishable from background is given by $\sigma_{\text {rescaled }} \times \mathcal{A}_{R P V}$. So a mass point in the $m_{T}-m_{\eta}$ plane is excluded if $\sigma_{\text {rescaled }}$ is greater than the observed $95 \%$ upper bound on the cross section $p p \rightarrow \tilde{g} \tilde{g}$ for a given value of $m_{T}\left(m_{T}=m_{\tilde{g}}\right)$ obtained by the search.

To understand the main features of the exclusion regions we obtained, it is instructive to look first at the ratio of acceptance $\frac{\mathcal{A}_{\text {our }}}{\mathcal{A}_{R P V}}$, which is shown in figure 3 as a function of the scalar mass for various fixed top partner masses. If $m_{\eta} \gg m_{T}$, then the topology of our model is the same as the RPV gluino decaying to three SM jets and we would expect $\frac{\mathcal{A}_{\text {our }}}{\mathcal{A}_{R P V}} \sim 1$. In this case the bound on $m_{T}$ can be found by simply comparing $\sigma(p p \rightarrow \bar{T} T)$ with the limit obtained by the CMS Collaboration [49]. However, if $m_{\eta} \lesssim m_{T}$, the scalar $\eta$ is on-shell and the topology is different from the RPV gluino. In particular, the distribution of the $D_{[3,2]}^{2}$ variable changes significantly as $\eta$ goes on-shell and becomes strongly dependent on the mass difference $m_{T}-m_{\eta}$. This is shown in figure 4 where distributions of the $D_{[3,2]}^{2}$ variable is shown for a fixed $m_{T}=900 \mathrm{GeV}$ and several scalar masses. ${ }^{2}$ We find that even with such different distributions, the efficiency of the search remains high in most of the on-shell $\eta$ parameter space because the cut applied on $D_{[3,2]}^{2}$ is relatively high, with the exceptions being for $m_{\eta} \approx m_{T}$ and $m_{T} \gg m_{\eta}$. The $D_{[3,2]}^{2}$ distributions for the RPV signal are extremely similar to case where $m_{\eta} \gg m_{T}$ as one would expect which we have alluded to before during the discussion of figure 3 .

These regions of low efficiencies can be understood as follows: when the mass splitting between $T$ and $\eta$ is small, the jet from the $T \rightarrow j \eta$ decay is soft, resulting in a decrease in $\mathcal{A}_{\text {our }}$ from the requirement of six hard jets. This topology however still has a di-jet signature, and can be probed by the di-jet search discussed in the next section. If $m_{T} \gg m_{\eta}$, the scalars are produced with very high boost and the jets resulting from their decay $\eta \rightarrow j j$ will often merge into a single jet leading once again to reduced sensitivity, but also having a di-jet like topology.

Performing a grid search for all mass points in the $m_{T}-m_{\eta}$ plane with increments of $25 \mathrm{GeV}$, we obtain the exclusion regions shown by the blue shaded area of figure 5 . We see that the dips in figure 3 translate to holes in the sensitivity of the CMS search for $m_{T} \sim m_{\eta}$

\footnotetext{
${ }^{1}$ In our simulation we found the invariant distribution to be slightly skewed towards lower masses, see appendix A for more details of this discrepancy.

${ }^{2}$ Other $m_{T}$ values show similar behaviour.
} 


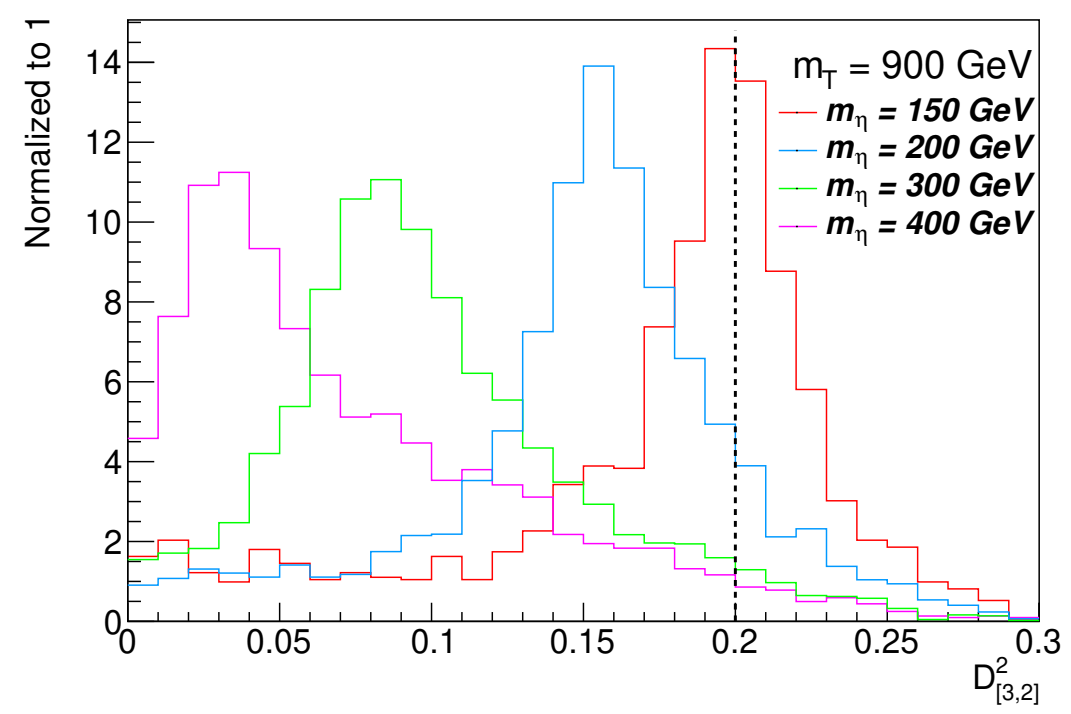

(a)

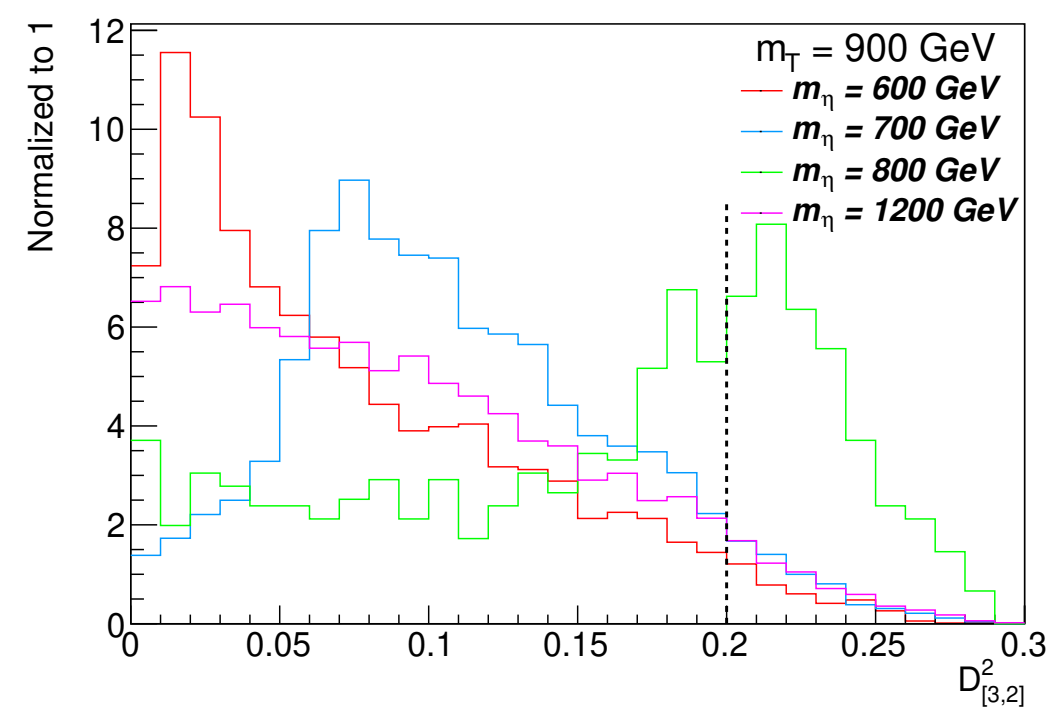

(b)

Figure 4. The $D_{[3,2]}^{2}$ variable distributions for signal triplets with top partner mass of $900 \mathrm{GeV}$ and various scalar masses. The black dashed line represent the cut placed on $D_{[3,2]}^{2}$ (accepting events below this value) for this particular top partner mass. 


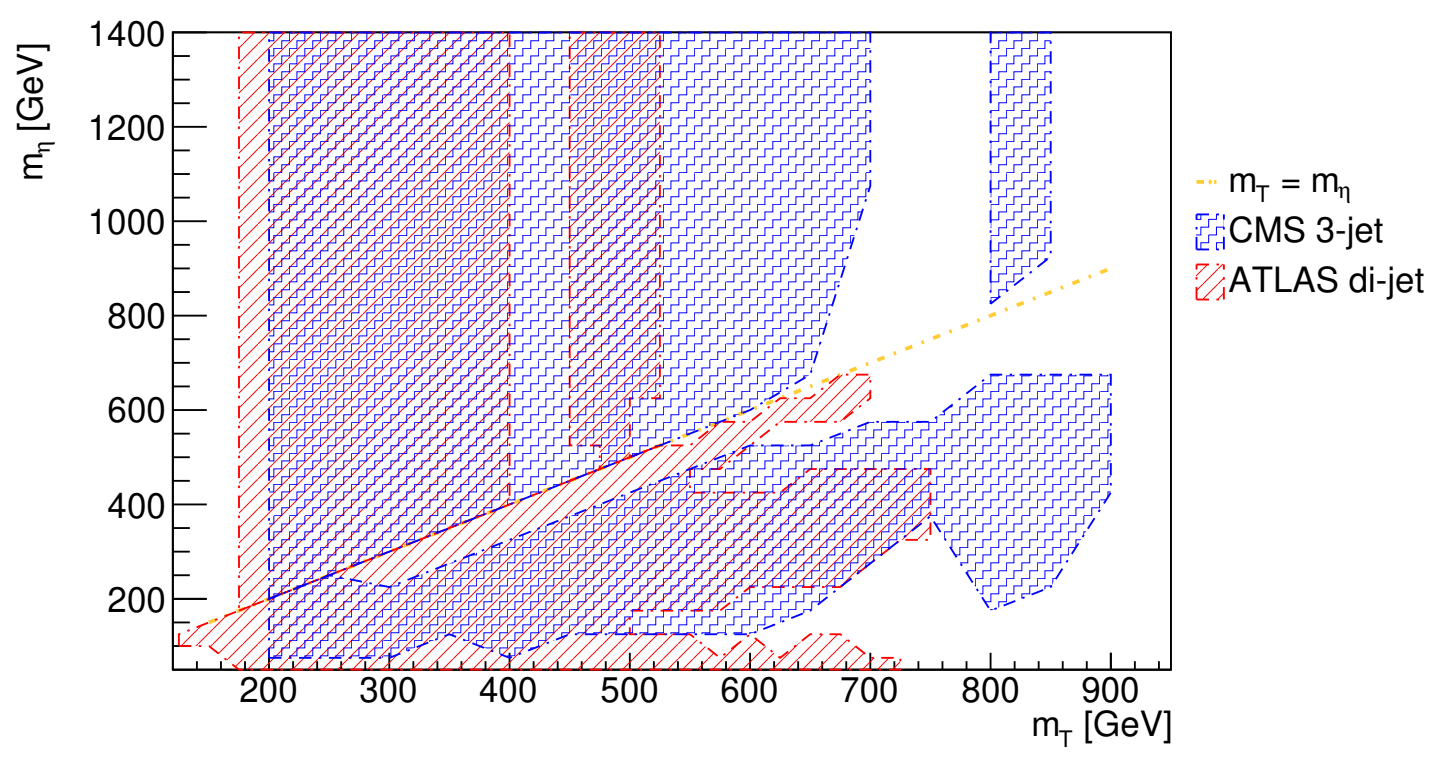

Figure 5. The CMS and ATLAS bounds on the fully hadronic decay mode (to light jets) of the top partner. The shaded regions are excluded by the current searches conducted at the LHC $[49,50]$.

and for $m_{\eta} \ll m_{T}$. We also see that the bounds in the bulk on-shell region $m_{T} \gtrsim m_{\eta}$ are stronger than the off-shell region $m_{T} \lesssim m_{\eta}$. The isolated exclusion near $m_{T} \sim 800 \mathrm{GeV}$ in the off-shell region can be attributed to a downward fluctuation of the background in that region.

\subsection{ATLAS pair-produced di-jet resonances}

As mentioned in the previous section, searches that look for pair-produced di-jet resonances can constrain this scenario in the region where $\eta$ is on-shell. ${ }^{3}$ It is especially useful when $T$ and $\eta$ are close in mass or the scalar is very light as the three-jet search is not sensitive in those regions. Such a search was conducted by ATLAS at $\sqrt{s}=13 \mathrm{TeV}$ with an integrated luminosity of $36.7 \mathrm{fb}^{-1}$ [50]. It explores coloured resonances that are pair-produced and that each decay to two jets, giving rise to a four-jet final state. The results of the analysis are interpreted in a simplified R-parity violating SUSY model where the top squark is the lightest supersymmetric particle and decays promptly into two quarks $\left(\tilde{t} \rightarrow \bar{q}_{j} \bar{q}_{k}\right)$. The search explores the region $100 \mathrm{GeV}<m_{\tilde{t}}<800 \mathrm{GeV}$ and excludes top squark masses in the range $100 \mathrm{GeV}<m_{\tilde{t}}<410 \mathrm{GeV}$ at $95 \% \mathrm{CL}$. The list of selection criteria is given in table 3 in the appendix. Since it is expected that the resonances are produced with high transverse momentum, their decay products will be located close to each other. As such, the four leading jets are paired using an angular distance:

$$
\Delta R_{\min }=\min \left\{\sum_{i=1}^{2}\left|\Delta R_{i}-1\right|\right\},
$$

\footnotetext{
${ }^{3}$ We will show later that this search also has significant sensitivity in the off-shell region.
} 
where $\Delta R_{i}=\sqrt{\Delta \phi_{i}^{2}+\Delta \eta_{i}^{2}}$ is the distance between the two jets in $i^{t h}$ pair. The two jet pairs selected must minimize $\Delta R_{\min }$ and satisfy the $\Delta R_{\text {min }}$ cut. Signal jets are expected to be produced in the central region so putting a cut on $\left|\cos \theta^{*}\right|$, where $\theta^{*}$ is the angle that either of the resonances makes with the beamline in the center-of-mass frame, is beneficial. Finally, the masses of the resonances are expected to be equal; hence, their invariant mass differences would be an ideal discriminant between signal and background. As such, the mass asymmetry $\left(\mathcal{A}_{m}\right)$, defined as:

$$
\mathcal{A}_{m}=\frac{\left|m_{1}-m_{2}\right|}{m_{1}+m_{2}}
$$

where $m_{1}$ and $m_{2}$ are the invariant masses of the two reconstructed di-jet pairs is required to be small. To set bounds, the ATLAS collaboration employed a modified frequentist approach using the $\mathrm{CL}_{s}[62-64]$ technique and a profile likelihood ratio as the test statistics. For each mass hypothesis, a counting experiment is performed in a window around the average mass of the two reconstructed resonances. The dominant source of background comes from QCD multijet production and is estimated directly from the data with a method that predicts both the normalization and the shape of the average di-jet mass distribution [50].

In order to recast the search, we simulated our particular model for the corresponding $m_{T}\left(m_{T}=m_{\tilde{t}}\right)$ for a fixed $m_{\eta}$. We computed the efficiency of the search for our model $\left(\epsilon_{\text {our }}\right)$, defined as the number of events satisfying all the cuts in table 3 plus an invariant mass window cut over the total number of simulated events. The number of signal events is then given as follows:

$$
N_{s}=\mathcal{L}_{\text {luminosity }} \times \sigma(p p \rightarrow \bar{T} T) \times \epsilon_{\text {our }} .
$$

We computed this number for various window mass cuts, corresponding to different stop masses considered in [50]. Taking the number of background and observed events and their uncertainties in the different windows from tables 3 and 5 of [50], we computed the confidence level:

$$
C L_{s}=\frac{P_{s+b}\left(X \leq X_{\mathrm{obs}}\right)}{P_{b}\left(X \leq X_{\mathrm{obs}}\right)}=\frac{C L_{s+b}}{C L_{b}} .
$$

Here $C L_{s+b}$ is the confidence level for excluding the possibility of simultaneous presence of signal and background while $C L_{b}$ is the probability that the test statistic is less than or equal to that observed in the data (assuming only the presence of background) [62-64]. Then a mass point is excluded at $95 \%$ confidence level if $\left(1-C L_{s}\right) \times 100 \%$ is greater than 95\%. The parameter space excluded by the ATLAS search is given by the red shaded region of figure 5 . It extends up to top partner masses of $\sim 750 \mathrm{GeV}$.

The di-jet search allows us to close some of the gaps that remain in the three-jet resonance search, namely the regions where $m_{\eta} \approx m_{T}$ or $m_{\eta} \ll m_{T}$. The gap in di-jet exclusion curve for $400 \mathrm{GeV} \leq m_{T} \leq 425 \mathrm{GeV}$ and $m_{T}<m_{\eta}$ can be explained by a downward fluctuation in the background in that region as illustrated in figure 9 (a) of the ATLAS search [50].

While the ATLAS search is primarily designed for di-jet topologies we also find reasonably good efficiency in the off-shell region where there is in fact no di-jet resonance, placing an exclusion up to $m_{T} \leq 525 \mathrm{GeV}$ in that region. In particular, one would expect 


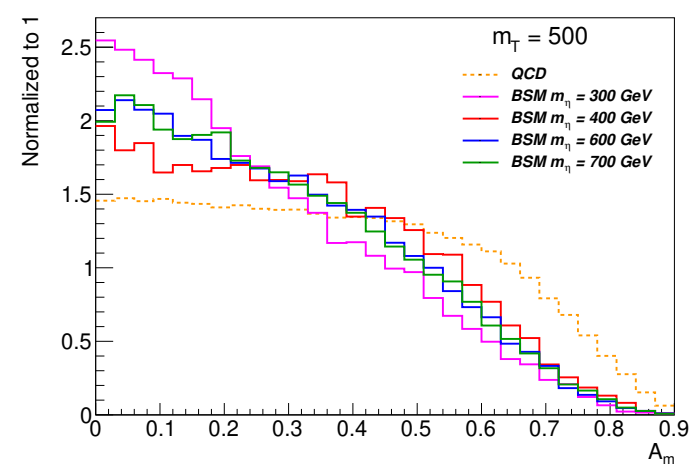

(a) Passing only $p_{T}$ cut.

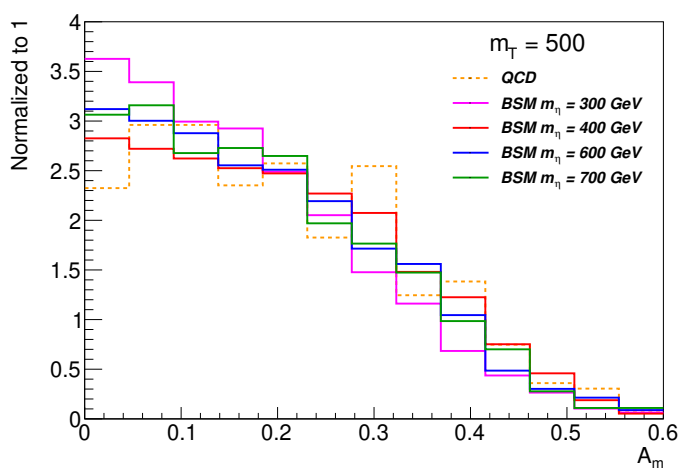

(b) Passing $p_{T}$ and $\Delta R_{\min }$ cuts.

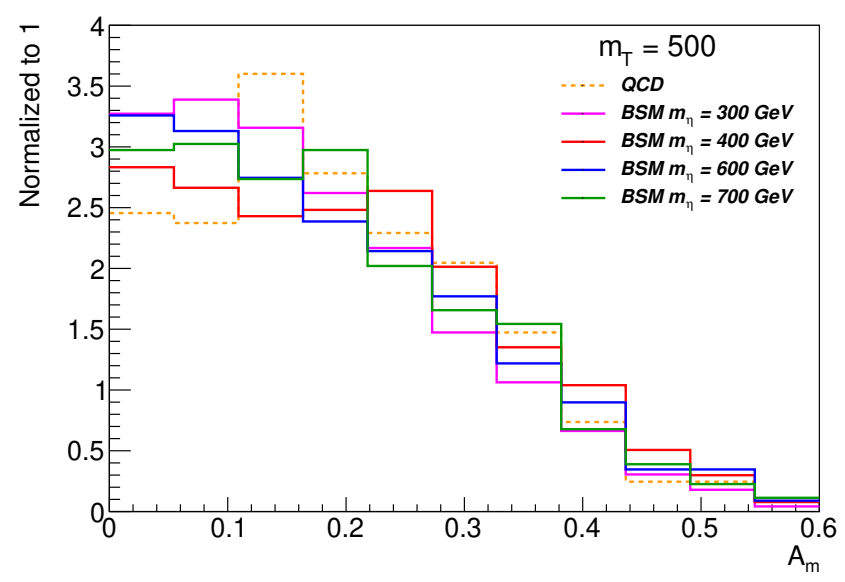

(c) Passing all but $\mathcal{A}_{m}$ cuts.

Figure 6. The $\mathcal{A}_{m}$ spectrum passing the various selection criteria as highlighted in the ATLAS search [50] for $m_{T}=500 \mathrm{GeV}$. The distributions of the (a) are for events passing only the $p_{T}$ requirement, the (b) satisfies both $p_{T}$ and $\Delta R_{\text {min }}$ requirements, and the (c) have all except $\mathcal{A}_{m}$ cuts applied.

the $A_{m}$ cut in eq. 2.5 would be very inefficient when there is no 2 -jet resonance. However, we find that the $\Delta R_{\min }$ and $\left|\cos \left(\theta^{*}\right)\right|$ selection criteria sculpt the $A_{m}$ distribution which becomes similar for the on-shell and off-shell cases. This is shown in figure 6 where $A_{m}$ is plotted after various cuts.

For very light top partner masses $m_{T} \lesssim 200 \mathrm{GeV}$, searches at previous experiments may be sensitive. For example, a three-jet hadronic resonance search was performed at $\sqrt{s}=1.96 \mathrm{GeV}$ at the Collider Detector at Fermilab (CDF) [65] and excluded gluinos below $144 \mathrm{GeV}$. Multijet searches performed at LEP has excluded the neutralino decaying to three jets for masses up to about $100 \mathrm{GeV}[66,67]$. There does remain a gap for $m_{T} \simeq m_{t}$ near the top quark. It may be possible to exclude this region of parameter space using the measurement of the all hadronic top quark decay [68-72], but a detailed analysis is beyond the scope of this work. 


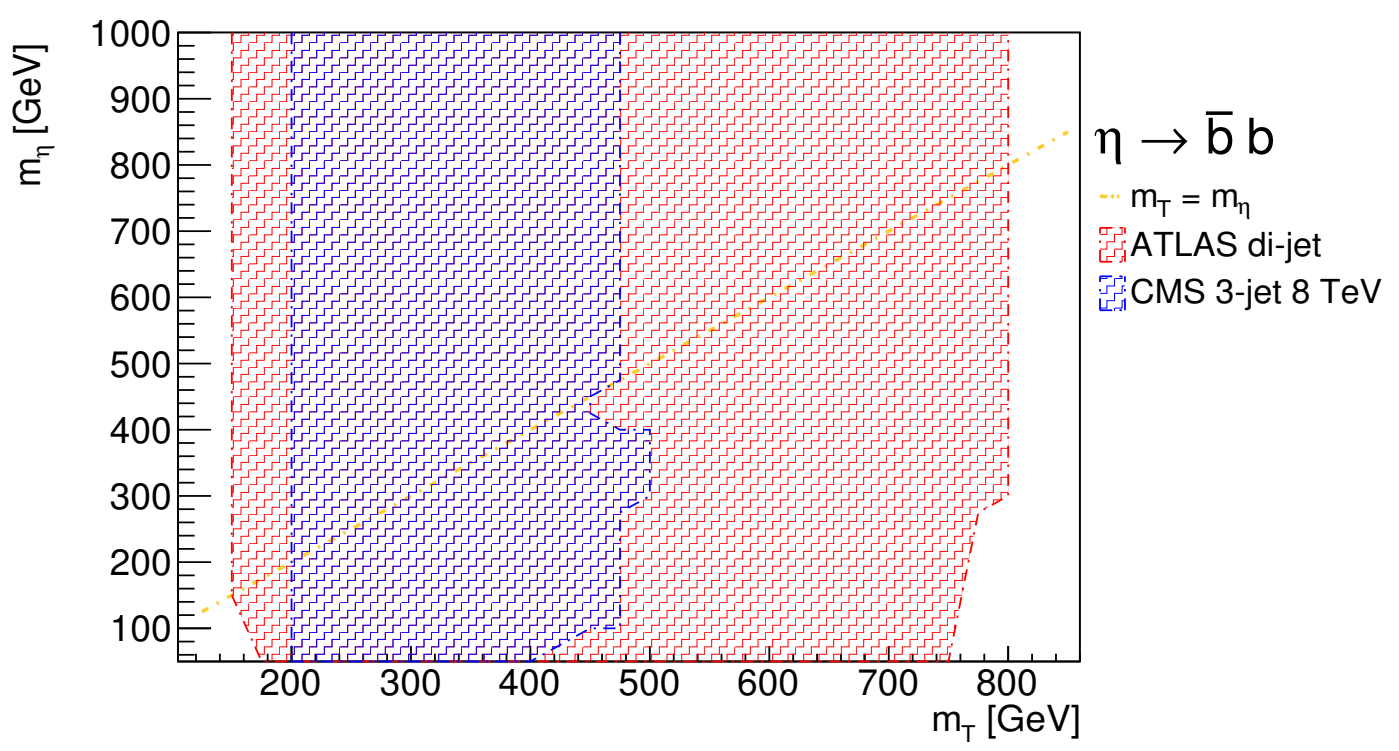

Figure 7. The CMS and ATLAS bounds on the fully hadronic decay mode (to heavy flavour jets) of the top partner. The shaded regions are excluded by the current searches conducted at the LHC $[50,74]$.

\section{Heavy flavour scenario}

In this section we study the case where the scalar $\eta$ decays to two $b$-jets: $p p \rightarrow T \bar{T} \rightarrow$ $j j \eta \eta \rightarrow 2 j 4 b$. This decay topology is what one would expect if, for example, the $\eta$ coupled to fermions proportionally to their masses and was lighter than twice the top mass. In principle such a final state allows for better discrimination from QCD multijet events. However, we find that existing searches do not give much stronger constraints. We considered the fully hadronic ATLAS R-parity-violating multijet searches [59] and [73] performed at $\sqrt{s}=8 \mathrm{TeV}$ and $13 \mathrm{TeV}$ respectively, the $13 \mathrm{TeV}$ ATLAS di-jet search [50] and the heavy flavour three-jet resonance CMS search [74] conducted at $\sqrt{s}=8 \mathrm{TeV}$. All of these searches have a $b$-tagging requirement in some of their signal regions, but only the CMS three-jet resonance and the ATLAS di-jet searches were found to place a limit on this scenario. The selection criteria for the heavy flavour three-jet resonance CMS search ${ }^{4}$ are presented in table 4 (in the appendix) and the limits on the parameter space are computed using the same method we used to recast [49] (see equation (2.3)). The region of parameter space excluded at $95 \%$ confidence level by this particular CMS search is presented by the blue shaded region of figure 7 .

We also recasted the ATLAS di-jet search [50], which requires at least two b-tagged jets in addition to the window cut around the average invariant mass of the di-jets with additional selection criteria given in table 3 (in the appendix). Computing the exclusion limits using the $\mathrm{CL}_{s}$ method [62-64], we obtain the red shaded region in figure 7 . The presence of at least two $b$-jet in the reconstructed resonances reduces the combinatoric

\footnotetext{
${ }^{4}$ The search also requires at least 6 jets and at least one $b$-jet.
} 
background coming from the two jet pairing. Hence, the search often selects the di-jet pairs corresponding to the scalar resonance, making the search more effective in both onshell and off-shell regions in comparison to the light jet scenario.

The searches considered in section 2 do not apply $b$-tags, but they also do not veto events with $b$-jets, so the exclusion regions shown in figure 5 also apply to the $b$-rich scenario considered in this section. Therefore, comparing figures 5 and 7 , we see that adding $b$ tagging only provides new exclusions for $m_{T}$ between about 700 and $800 \mathrm{GeV}$ and some values of $m_{\eta}$. The ATLAS search [50], however, only investigates $m_{\tilde{t}}$ up to $800 \mathrm{GeV}$ which is almost entirely excluded. ${ }^{5}$ So in principle, the ATLAS di-jet search could have excluded larger $m_{T}$. Due to the steeply decreasing cross section, shown in figure 2, it is unlikely that extending the search would have resulted in an exclusion region significantly above $800 \mathrm{GeV}$.

The $\sqrt{s}=13 \mathrm{TeV}$ three light jets resonance search from CMS analyzed in section 2.1 places the strongest constraints on three-jet resonances. If this search included a signal region with $b$-tagging, it could also improve constraints on the $b$-rich scenario. Here we give a rough appraisal of the potential improvement. First, we estimate the total number of QCD multijet events by simulating $p p \longrightarrow j j j j$ using MadGraph5 interfaced with Pythia8 and Delphes (more detail on the simulation is given in appendix D). The acceptance $\left(\mathcal{A}_{Q C D}\right)$ is obtained by applying all the selection criteria for the three-jet CMS search and we approximate the number of background events to be: $b=\mathcal{L}_{\text {luminosity }} \times \sigma(p p \rightarrow j j j j) \times \mathcal{A}_{Q C D}$. We set the minimum parton level $p_{T}$ for the simulated QCD events to be $100 \mathrm{GeV}$ in order to have enough events for our rough approximation. In a similar way we compute $s$, the number of signal events using simulation of our model. We then compare the $s / \sqrt{b}$ values obtained without $b$-tagging to the case where with $2 b$-tags included in the cuts, and find that $s / \sqrt{b}$ is roughly a factor of three larger. The three-jet CMS search excludes $m_{T} \lesssim 900 \mathrm{GeV}$ for the light jet case, so our rough estimate is that including a $b$-tag requirement could exclude the $b$-jet topology up to $m_{T} \sim 1050 \mathrm{GeV}$ as the ratio of top partner cross section for $900 \mathrm{GeV}$ and 1050 top partner masses is also $\sim 3$. Requiring at least 4 $b$-tags could further increase the expected exclusion to $\sim 1300 \mathrm{GeV}$. We summarize the improvements for different numbers of $b$-tags in table 1.

In a similar manner, we can roughly estimate the effect of extending the ATLAS dijet search to higher masses by computing $R \equiv \sigma_{\text {rescaled }} / \sigma_{\text {obs }}$ at the cut off region $\left(m_{T}=\right.$ $800 \mathrm{GeV}$ ) where $\sigma_{\text {obs }}$ is the observed $95 \%$ CL from ATLAS di-jet search for a given mass and $\sigma_{\text {rescaled }}$ is the rescaled cross section as defined in equation (2.3). The corresponding value for $R$ is approximately 3 which points to a limit of $\sim m_{T}=950$ as the ratio of cross section for top partner masses of 950 and $800 \mathrm{GeV}$ is $\sim 3$.

\section{Conclusion}

Although there has been a significant experimental search program for top partner pair production with $t h, b W$ and $t Z$ decay modes, to our knowledge there has not been any studies to explore the all light jet decay mode. In this work we have recasted the latest available LHC searches that can impose significant constraints on the parameter space of

\footnotetext{
${ }^{5}$ With the exception of the very light scalars for $m_{T} \leq 150 \mathrm{GeV}$ and low scalar masses for $m_{T} \geq 775 \mathrm{GeV}$.
} 


\begin{tabular}{|c|ccc|}
\hline B-tagging requirement & $\frac{s}{\sqrt{b}}$ & Improvement & Mass Sensitivity $[\mathrm{GeV}]$ \\
\hline$N_{b} \geq 0$ & 0.31 & - & 900 \\
\hline$N_{b} \geq 1$ & 0.58 & 1.86 & 1000 \\
$N_{b} \geq 2$ & 1.04 & 3.33 & 1050 \\
$N_{b} \geq 3$ & 2.30 & 8.36 & 1200 \\
$N_{b} \geq 4$ & 4.66 & 14.89 & 1300 \\
\hline
\end{tabular}

Table 1. The approximate improvement in $s / \sqrt{b}$ and mass reach as a function of increasing number of $b$-tags, $N_{b}$. The first line is the actual limit, while the subsequent lines are estimated potential improvements. The $s / \sqrt{b}$ values are computed at the cut off region of $m_{T}=900 \mathrm{GeV}$ for the three-jet CMS search [49] with $35.9 \mathrm{fb}^{-1}$.

models where the top partner decays to light jets. Our results are shown in figures 5 and 7 for models with final states containing only light jets and for final states containing $4 b$-jets respectively. Top partner masses are generally excluded up to $m_{T} \sim 900 \mathrm{GeV}$, but there are a few gaps in the $m_{T}-m_{\eta}$ plane for lighter $m_{T}$. Because the three-jet resonance search we recast focused on a resonance that decays through an off-shell scalar, it might be possible to obtain better limit in the on-shell $\eta$ region by designing a search specifically for that topology. Furthermore we found that existing searches do not provide significantly better constraints for the case where the final states contain $b$-jet, but those limits could be improved by adding $b$-tagging requirements to the $13 \mathrm{TeV}$ three-jet resonance search.

\section{Acknowledgments}

We thank Jack Collins and Jesse Thaler for helpful discussions. This work was supported in part by the Natural Sciences and Engineering Research Council of Canada (NSERC).

\section{A CMS three jet resonance search}

The $13 \mathrm{TeV}$ CMS search [49] requires an event to contain at least six jets with $|\eta|<2.4$. The jet reconstruction is performed using the anti- $k_{t}$ algorithm [75] with a radius parameter of $R=0.4$. The list of all the selection criteria used in this particular search is given in table 2. The analysis employs the jet-ensemble technique [57, 65], which takes the six highest $p_{T}$ jets in a given event and group them into 20 unique triplets. For signal, at most 2 of these triplets per event corresponds to the pair produced gluino decay while the rest contributes to combinatoric background which are referred to as "incorrect" triplets. Consequently the acceptance is defined as the ratio of the correct triplet over the total number of triplets (20) in the event. Furthermore, an event-level variable $D_{[(6,3)+(3,2)]}^{2}$ is defined in order to characterize the angular spread of the six constituent jets insides a pair of triplets. The six-jet distance measure is defined as:

$$
D_{[(6,3)+(3,2)]}^{2}=\sum_{i<j<k}\left(\sqrt{\hat{m}(6,3)_{i j k}^{2}+D_{[3,2], i j k}^{2}}-\frac{1}{\sqrt{20}}\right)^{2},
$$




\begin{tabular}{|cccccccc|}
\hline $\begin{array}{c}\text { Gluino mass } \\
\text { range }[\mathrm{GeV}]\end{array}$ & Jet $p_{T}[\mathrm{GeV}]$ & $H_{T}[\mathrm{GeV}]$ & $\begin{array}{c}\text { Sixth Jet } \\
p_{T}[\mathrm{GeV}]\end{array}$ & $D_{[(6,3)+(3,2)]}^{2}$ & $A_{m}$ & $\Delta[\mathrm{GeV}]$ & $D_{[3,2]}^{2}$ \\
\hline $200-400$ & $>30$ & $>650$ & $>40$ & $<1.25$ & $<0.25$ & $>250$ & $<0.05$ \\
$400-700$ & $>30$ & $>650$ & $>50$ & $<1.00$ & $<0.175$ & $>180$ & $<0.175$ \\
$700-1200$ & $>50$ & $>900$ & $>125$ & $<0.9$ & $<0.15$ & $>20$ & $<0.2$ \\
$1200-2000$ & $>50$ & $>900$ & $>175$ & $<0.75$ & $<0.15$ & $>-120$ & $<0.25$ \\
\hline
\end{tabular}

Table 2. The list of selection criteria with the direction of the cuts and the mass ranges analyzed by the CMS search [49].

where $\hat{m}(6,3)_{i j k}^{2}=\frac{m_{i j k}^{2}}{4 \cdot m_{i j k l m n}^{2}+6 \sum_{i} m_{i}^{2}}$ with $i, j, k, l, m, n \in\{1,2, \ldots, 6\}$ and $m_{i j k l m n}$ the invariant mass of the six highest $p_{T}$ jets. For a new particle decaying to three-jets, the jets produced would be uniformly distributed in a detector resulting in $\hat{m}(6,3)_{i j k}^{2}$ approximately $1 / 20$. While the jets from the QCD are usually grouped together giving $\hat{m}(6,3)_{i j k}^{2}$ close to zero or one.

Furthermore one of the most efficient cut for the three-jet resonance is the "Delta cut" defined as:

$$
M_{j j j}<\sum_{i=1}^{3} p_{T}^{i}-\Delta,
$$

where $M_{j j j}$ is the invariant mass of the triplet and $\Delta$ is an adjustable parameter. The parameter $\Delta$ is determined in each signal region by optimizing the signal significance $\alpha=$ $s / \sqrt{s+b}$. This particular selection criteria can be understood due to the observation of the linear correlation of the triplet invariant mass with scalar sum of the transverse momentum for the QCD background. While the triplet invariant mass of the correctly combined signal triplets is unchanged by varying $p_{T}$ since $M_{j j j}$ is fixed. Consequently, this not only reduces the QCD multijet background but the combinatoric background raising from the incorrectly combined signal triplets as well. Finally, the mass asymmetry variable is defined as:

$$
A_{m}=\frac{\left|m_{i j k}-m_{l m n}\right|}{m_{i j k}+m_{l m n}},
$$

where $m_{i j k}$ is invariant mass of the triplet. This variable has discriminating power between signal and background since the signal triplets are expected to be close each other in mass but not the background.

When trying to reproduce the $13 \mathrm{TeV}$ CMS search [49] we encountered some difficulites. The CMS collaboration paper contains $M_{j j j}$ distributions for their signal topologies reproduced in the top panels of figure 8. The shapes of the signals appear as perfect Gaussians centered around the gluino mass. However, our simulations of the RPV model result in an invariant mass peak that is slightly shifted below the true mass points and is asymmetric about the peak with a longer tail at lower invariant mass. We show the signal distributions for two different gluino masses in the bottom panel of figure 8 both with and without detector simulation. Furthermore, it is stated in [49] that the invariant mass distribution of the incorrectly combined signal triplets (the combinatoric background) is similar to the multijet background; however, we find them to be different. There is also an ambiguity in 


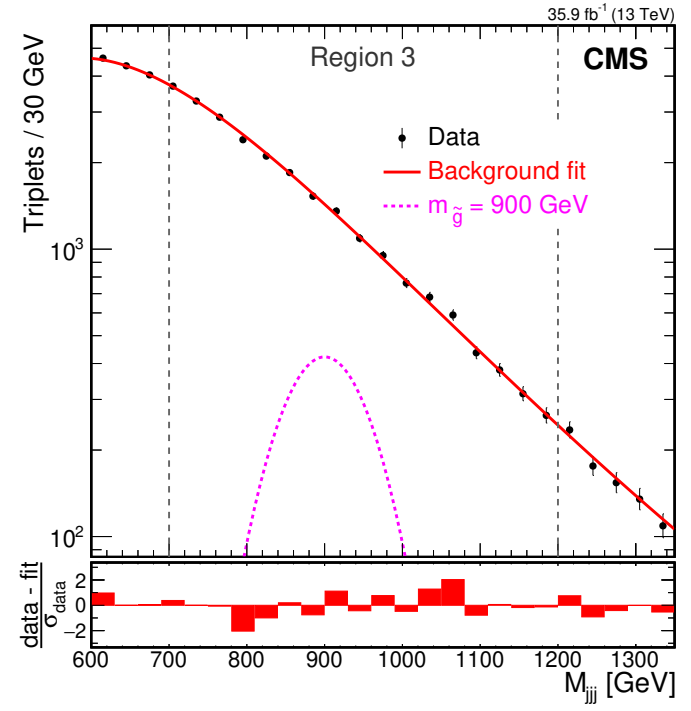

(a)

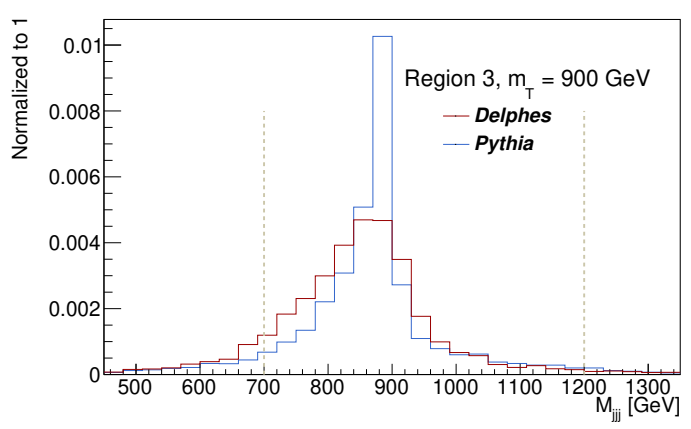

(c)

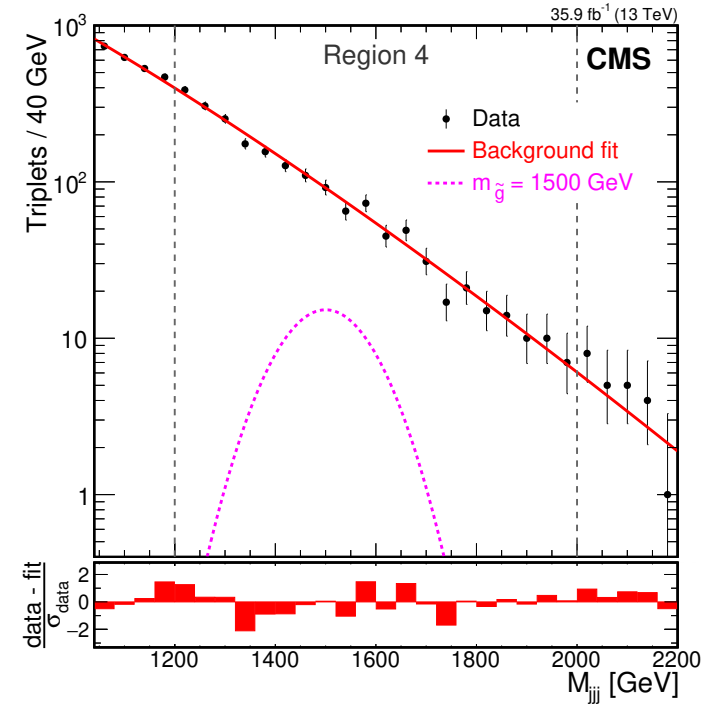

(b)

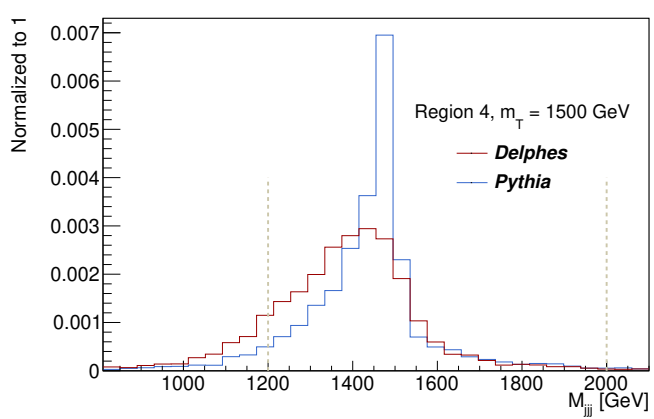

(d)

Figure 8. Mass distributions for two of the mass regions. The distributions in figure (a) and (b) are produced by CMS [49] while the bottom figures are the corresponding ones for $m_{\eta}=5 \mathrm{TeV}$.

the definition of the acceptance in the case where more than two triplets in an event satisfy all the selection criteria. Finally, [49] refers to Monte Carlo simulations of the QCD background, but the work does not specify how the QCD samples are generated. More details about the procedures for computing the signal efficiency and simulating the background would be helpful for future studies and recasts.

\section{B ATLAS di-jet resonance search}

Similar to the three-jet CMS search, this di-jet search [50] also reconstructs the jet candidates using an anti- $k_{t}$ algorithm with a radius parameter of 0.4 . The complete list of cuts is displayed in table 3. The average mass of the two reconstructed resonances is expected to peak around the mass of the resonance being searched for. The average mass,

$$
m_{\mathrm{avg}}=\frac{1}{2}\left(m_{1}+m_{2}\right),
$$




\begin{tabular}{|cccc|}
\hline Jet $p_{T}[\mathrm{GeV}]$ & $\mathcal{A}_{m}$ & $\left|\cos \left(\theta^{*}\right)\right|$ & $\Delta R_{\min }$ \\
\hline$>120$ & $<0.05$ & $<0.3$ & $<-0.002 \cdot\left(\frac{m_{\text {avg }}}{\mathrm{GeV}}-225\right)+0.72, \quad$ if $m_{\text {avg }} \leq 225 \mathrm{GeV}$ \\
& & & $<+0.0013 \cdot\left(\frac{m_{\text {avg }}}{\mathrm{GeV}}-225\right)+0.72, \quad$ if $m_{\text {avg }}>225 \mathrm{GeV}$ \\
\hline
\end{tabular}

Table 3. The list of selection criteria with the direction of the cut for the ATLAS di-jet search [50].

\begin{tabular}{|ccccc|}
\hline Mass Range $[\mathrm{GeV}]$ & $\Delta[\mathrm{GeV}]$ & $p_{T, j}^{4 t h}[\mathrm{GeV}]$ & $p_{T, j}^{6 t h}[\mathrm{GeV}]$ & Sphericity \\
\hline $200-600$ & $>110$ & $>80$ & $>60$ & - \\
$600-1500$ & $>110$ & $>110$ & $>110$ & $>0.4$ \\
\hline
\end{tabular}

Table 4. The selection criteria with the direction of the cut for the CMS heavy flavour search performed at $\sqrt{s}=8 \mathrm{TeV}[74]$.

is thus required to be inside of a window around the searched for mass, with the width of the window varying from 10 to $100 \mathrm{GeV}$ and is given in tables 3 and 5 of [50]. In order to recast this particular search, the RPV top squarks were pair produced with radiation of up to two additional partons. The merging with parton shower was done using the MLM [76] prescription with a merging scale set to $1 / 4$ of the top squark mass. In addition, all the SUSY particles except top squark were decoupled by setting their masses to $5 \mathrm{TeV}$.

\section{CMS three jet resonance search at $\sqrt{s}=8 \mathrm{TeV}$}

The work of [74] is an earlier version of the three-jet CMS search [49] performed at the center-of-mass energy of $8 \mathrm{TeV}$. The jet candidates are constructed using an anti- $k_{t}$ algorithm with a radius parameter of 0.5 . The search considers two scenarios, first when the gluino decays into light flavour jets and secondly when it decays to a $b$-jet and two light flavour jets. The latter case requires the existence of at least one bottom quark jet in the resonance decay products. Besides the usual $p_{T}$ and $\Delta$ variables requirements described in appendix A, event shape information is exploited. Typically in the high mass region, the signal events have a more spherical shape than the background (which generally contain back to back jets thus more linear shape) [74]. Consequently, the sphericity variable is defined as,

$$
S=\frac{3}{2}\left(\lambda_{2}+\lambda_{3}\right), \quad \lambda_{1} \geq \lambda_{2} \geq \lambda_{3}
$$

where $\lambda$ 's are the eigenvalues of the sphericity tensor,

$$
S^{\alpha \beta}=\frac{\sum_{i} p_{i}^{\alpha} p_{i}^{\beta}}{\sum_{i}\left|p_{i}\right|^{2}}, \quad \alpha, \beta=\mathrm{x}, \mathrm{y}, \mathrm{z},
$$

where $\alpha$ and $\beta$ label separate jets, and the sphericity $S$ is calculated using all jets in each event. The complete list of selection criteria are shown in table 4 . 


\begin{tabular}{|cc|}
\hline$p_{T, \min }(j)$ Generator Level $[\mathrm{GeV}]$ & $\sigma_{4 j}[\mathrm{pb}]$ \\
\hline 20 & $1.79 \times 10^{7}$ \\
60 & $7.64 \times 10^{4}$ \\
100 & $4.68 \times 10^{3}$ \\
200 & $7.216 \times 10^{1}$ \\
\hline
\end{tabular}

Table 5. Four partonic hard jets production cross section using MadGraph5 at $\sqrt{s}=13 \mathrm{TeV}$ with various minimum parton level cut.

\section{Multijet background}

The principal background for our signal arises from the QCD multijet events. Other SM processes have negligible contributions, and we have performed simulations of $\bar{t} t$ events to confirm that their rates are indeed very small. The QCD multijet background is very large as one can observe from the crude cross section estimates shown in table 5 (similar results were obtained using Sherpa [77]). The QCD multijet events were obtained by simulating $p p \rightarrow j j j j$ using MadGraph5 interfaced with Pythia8 and Delphes. The cross sections are orders of magnitude larger than the pair production cross section for top partner as displayed in figure 2. In our simulations, we require each of the four partons to have $p_{T}>p_{T, \min }(j)=100 \mathrm{GeV}$ in order to make sure enough events satisfy all the selection requirements for our recasted searches. The minimum parton level $p_{T}$ is well below the detector level jet $p_{T}$ requirement of $125 \mathrm{GeV}$, given in table 2 , at the cut off region $\left(m_{T}=900 \mathrm{GeV}\right)$, so it does not affect our analysis.

Open Access. This article is distributed under the terms of the Creative Commons Attribution License (CC-BY 4.0), which permits any use, distribution and reproduction in any medium, provided the original author(s) and source are credited.

\section{References}

[1] ATLAS collaboration, Observation of a new particle in the search for the Standard Model Higgs boson with the ATLAS detector at the LHC, Phys. Lett. B 716 (2012) 1 [arXiv: 1207.7214] [INSPIRE].

[2] CMS collaboration, Observation of a new boson at a mass of $125 \mathrm{GeV}$ with the CMS experiment at the LHC, Phys. Lett. B 716 (2012) 30 [arXiv:1207.7235] [InSPIRE].

[3] R. Contino, L. Da Rold and A. Pomarol, Light custodians in natural composite Higgs models, Phys. Rev. D 75 (2007) 055014 [hep-ph/0612048] [INSPIRE].

[4] D. Marzocca, M. Serone and J. Shu, General composite Higgs models, JHEP 08 (2012) 013 [arXiv: 1205.0770] [INSPIRE].

[5] O. Matsedonskyi, G. Panico and A. Wulzer, Light top partners for a light composite Higgs, JHEP 01 (2013) 164 [arXiv:1204.6333] [INSPIRE].

[6] A. De Simone, O. Matsedonskyi, R. Rattazzi and A. Wulzer, A first top partner hunter's guide, JHEP 04 (2013) 004 [arXiv: 1211.5663] [INSPIRE]. 
[7] B. Bellazzini, C. Csáki and J. Serra, Composite Higgses, Eur. Phys. J. C 74 (2014) 2766 [arXiv: 1401.2457] [INSPIRE].

[8] N. Arkani-Hamed, A.G. Cohen, E. Katz and A.E. Nelson, The littlest Higgs, JHEP 07 (2002) 034 [hep-ph/0206021] [INSPIRE].

[9] M. Schmaltz and D. Tucker-Smith, Little Higgs review, Ann. Rev. Nucl. Part. Sci. 55 (2005) 229 [hep-ph/0502182] [INSPIRE].

[10] ATLAS collaboration, Search for pair production of vector-like top quarks in events with one lepton, jets, and missing transverse momentum in $\sqrt{s}=13 \mathrm{TeV}$ pp collisions with the ATLAS detector, JHEP 08 (2017) 052 [arXiv:1705.10751] [INSPIRE].

[11] CMS collaboration, Search for pair production of vector-like $T$ and $B$ quarks in single-lepton final states using boosted jet substructure in proton-proton collisions at $\sqrt{s}=13 \mathrm{TeV}$, JHEP 11 (2017) 085 [arXiv:1706.03408] [INSPIRE].

[12] CMS collaboration, Search for pair production of vector-like quarks in the $b W \bar{b} W$ channel from proton-proton collisions at $\sqrt{s}=13 \mathrm{TeV}$, Phys. Lett. B 779 (2018) 82 [arXiv: 1710.01539] [INSPIRE].

[13] ATLAS collaboration, Search for pair production of up-type vector-like quarks and for four-top-quark events in final states with multiple b-jets with the ATLAS detector, JHEP 07 (2018) 089 [arXiv: 1803.09678] [INSPIRE].

[14] CMS collaboration, Search for vector-like $T$ and $B$ quark pairs in final states with leptons at $\sqrt{s}=13 \mathrm{TeV}, \mathrm{JHEP} 08$ (2018) 177 [arXiv:1805.04758] [INSPIRE].

[15] ATLAS collaboration, Search for pair production of heavy vector-like quarks decaying into high-p $p_{T} W$ bosons and top quarks in the lepton-plus-jets final state in pp collisions at $\sqrt{s}=13 \mathrm{TeV}$ with the ATLAS detector, JHEP 08 (2018) 048 [arXiv:1806. 01762] [INSPIRE].

[16] ATLAS collaboration, Search for pair production of heavy vector-like quarks decaying into hadronic final states in pp collisions at $\sqrt{s}=13 \mathrm{TeV}$ with the ATLAS detector, Phys. Rev. D 98 (2018) 092005 [arXiv: 1808.01771] [INSPIRE].

[17] ATLAS collaboration, Combination of the searches for pair-produced vector-like partners of the third-generation quarks at $\sqrt{s}=13$ TeV with the ATLAS detector, Phys. Rev. Lett. 121 (2018) 211801 [arXiv: 1808.02343] [INSPIRE].

[18] CMS collaboration, Search for vector-like quarks in events with two oppositely charged leptons and jets in proton-proton collisions at $\sqrt{s}=13$ TeV, Eur. Phys. J. C 79 (2019) 364 [arXiv: 1812.09768] [INSPIRE].

[19] CMS collaboration, Search for pair production of vectorlike quarks in the fully hadronic final state, Phys. Rev. D 100 (2019) 072001 [arXiv:1906.11903] [INSPIRE].

[20] ATLAS collaboration, Search for pair and single production of new heavy quarks that decay to a $Z$ boson and a third-generation quark in pp collisions at $\sqrt{s}=8$ TeV with the ATLAS detector, JHEP 11 (2014) 104 [arXiv:1409.5500] [INSPIRE].

[21] ATLAS collaboration, Search for the production of single vector-like and excited quarks in the Wt final state in pp collisions at $\sqrt{s}=8 \mathrm{TeV}$ with the ATLAS detector, JHEP 02 (2016) 110 [arXiv: 1510.02664] [INSPIRE].

[22] ATLAS collaboration, Search for single production of vector-like quarks decaying into Wb in pp collisions at $\sqrt{s}=8 \mathrm{TeV}$ with the ATLAS detector, Eur. Phys. J. C 76 (2016) 442 [arXiv: 1602.05606] [INSPIRE]. 
[23] CMS collaboration, Search for single production of a heavy vector-like $T$ quark decaying to a Higgs boson and a top quark with a lepton and jets in the final state, Phys. Lett. $B 771$ (2017) 80 [arXiv: 1612.00999] [INSPIRE].

[24] CMS collaboration, Search for electroweak production of a vector-like quark decaying to a top quark and a Higgs boson using boosted topologies in fully hadronic final states, JHEP 04 (2017) 136 [arXiv:1612.05336] [INSPIRE].

[25] CMS collaboration, Search for single production of vector-like quarks decaying to a $Z$ boson and a top or a bottom quark in proton-proton collisions at $\sqrt{s}=13$ TeV, JHEP 05 (2017) 029 [arXiv: 1701.07409] [INSPIRE].

[26] CMS collaboration, Search for single production of vector-like quarks decaying into a $b$ quark and $a W$ boson in proton-proton collisions at $\sqrt{s}=13$ TeV, Phys. Lett. B 772 (2017) 634 [arXiv: 1701.08328] [INSPIRE].

[27] CMS collaboration, Search for single production of a vector-like $T$ quark decaying to a $Z$ boson and a top quark in proton-proton collisions at $\sqrt{s}=13$ TeV, Phys. Lett. $B 781$ (2018) 574 [arXiv:1708.01062] [INSPIRE].

[28] CMS collaboration, Search for single production of vector-like quarks decaying to a $b$ quark and a Higgs boson, JHEP 06 (2018) 031 [arXiv:1802.01486] [INSPIRE].

[29] ATLAS collaboration, Search for pair- and single-production of vector-like quarks in final states with at least one $Z$ boson decaying into a pair of electrons or muons in pp collision data collected with the ATLAS detector at $\sqrt{s}=13$ TeV, Phys. Rev. D 98 (2018) 112010 [arXiv: 1806.10555] [INSPIRE].

[30] CMS collaboration, Search for single production of vector-like quarks decaying to a top quark and a $W$ boson in proton-proton collisions at $\sqrt{s}=13$ TeV, Eur. Phys. J. C 79 (2019) 90 [arXiv: 1809.08597] [INSPIRE].

[31] A. Anandakrishnan, J.H. Collins, M. Farina, E. Kuflik and M. Perelstein, Odd top partners at the LHC, Phys. Rev. D 93 (2016) 075009 [arXiv:1506.05130] [InSPIRE].

[32] J.A. Aguilar-Saavedra, D.E. López-Fogliani and C. Muñoz, Novel signatures for vector-like quarks, JHEP 06 (2017) 095 [arXiv: 1705.02526] [INSPIRE].

[33] J.A. Aguilar-Saavedra, J. Alonso-González, L. Merlo and J.M. No, Exotic vectorlike quark phenomenology in the minimal linear $\sigma$ model, Phys. Rev. D 101 (2020) 035015 [arXiv: 1911.10202] [INSPIRE].

[34] J.H. Kim and I.M. Lewis, Loop induced single top partner production and decay at the LHC, JHEP 05 (2018) 095 [arXiv: 1803.06351] [INSPIRE].

[35] H. Alhazmi, J.H. Kim, K. Kong and I.M. Lewis, Shedding light on top partner at the LHC, JHEP 01 (2019) 139 [arXiv: 1808.03649] [INSPIRE].

[36] R. Benbrik et al., Signatures of vector-like top partners decaying into new neutral scalar or pseudoscalar bosons, JHEP 05 (2020) 028 [arXiv: 1907.05929] [INSPIRE].

[37] J.H. Kim, S.D. Lane, H.-S. Lee, I.M. Lewis and M. Sullivan, Searching for dark photons with maverick top partners, Phys. Rev. D 101 (2020) 035041 [arXiv: 1904.05893] [INSPIRE].

[38] M. Chala, Direct bounds on heavy toplike quarks with standard and exotic decays, Phys. Rev. D 96 (2017) 015028 [arXiv:1705.03013] [INSPIRE]. 
[39] N. Bizot, G. Cacciapaglia and T. Flacke, Common exotic decays of top partners, JHEP 06 (2018) 065 [arXiv: 1803.00021] [INSPIRE].

[40] G. Cacciapaglia, T. Flacke, M. Park and M. Zhang, Exotic decays of top partners: mind the search gap, Phys. Lett. B 798 (2019) 135015 [arXiv:1908.07524] [INSPIRE].

[41] ATLAS collaboration, Performance and calibration of the JetFitterCharm algorithm for c-jet identification, Tech. Rep. ATL-PHYS-PUB-2015-001, CERN, Geneva, Switzerland (2015).

[42] CMS collaboration, Identification of c-quark jets at the CMS experiment, Tech. Rep. CMS-PAS-BTV-16-001, CERN, Geneva, Switzerland (2016).

[43] CMS collaboration, Identification of heavy-flavour jets with the CMS detector in pp collisions at $13 \mathrm{TeV}, 2018$ JINST 13 P05011 [arXiv:1712.07158] [INSPIRE].

[44] ATLAS collaboration, Measurements of b-jet tagging efficiency with the ATLAS detector using $t \bar{t}$ events at $\sqrt{s}=13 \mathrm{TeV}$, JHEP 08 (2018) 089 [arXiv: 1805.01845] [INSPIRE].

[45] Y. Kats, M. McCullough, G. Perez, Y. Soreq and J. Thaler, Colorful twisted top partners and partnerium at the LHC, JHEP 06 (2017) 126 [arXiv: 1704.03393] [INSPIRE].

[46] H.K. Dreiner, An introduction to explicit R-parity violation, in Perspectives on supersymmetry. Volume 2, G.L. Kane ed., World Scientific, Singapore (2010), pg. 565 [hep-ph/9707435] [INSPIRE].

[47] B.C. Allanach, A. Dedes and H.K. Dreiner, R-parity violating minimal supergravity model, Phys. Rev. D 69 (2004) 115002 [Erratum ibid. 72 (2005) 079902] [hep-ph/0309196] [INSPIRE].

[48] R. Barbier et al., R-parity violating supersymmetry, Phys. Rept. 420 (2005) 1 [hep-ph/0406039] [INSPIRE].

[49] CMS collaboration, Search for pair-produced three-jet resonances in proton-proton collisions at $\sqrt{s}=13 \mathrm{TeV}$, Phys. Rev. D 99 (2019) 012010 [arXiv:1810.10092] [INSPIRE].

[50] ATLAS collaboration, A search for pair-produced resonances in four-jet final states at $\sqrt{s}=13$ TeV with the ATLAS detector, Eur. Phys. J. C 78 (2018) 250 [arXiv:1710.07171] [INSPIRE].

[51] A. Alloul, N.D. Christensen, C. Degrande, C. Duhr and B. Fuks, FeynRules $2.0-a$ complete toolbox for tree-level phenomenology, Comput. Phys. Commun. 185 (2014) 2250 [arXiv:1310.1921] [INSPIRE].

[52] J. Alwall et al., The automated computation of tree-level and next-to-leading order differential cross sections, and their matching to parton shower simulations, JHEP 07 (2014) 079 [arXiv: 1405.0301] [INSPIRE].

[53] T. Sjöstrand et al., An introduction to PYTHIA 8.2, Comput. Phys. Commun. 191 (2015) 159 [arXiv: 1410.3012] [INSPIRE].

[54] DELPHES 3 collaboration, DELPHES 3, a modular framework for fast simulation of a generic collider experiment, JHEP 02 (2014) 057 [arXiv:1307.6346] [INSPIRE].

[55] M. Cacciari, G.P. Salam and G. Soyez, FastJet user manual, Eur. Phys. J. C 72 (2012) 1896 [arXiv: 1111.6097] [INSPIRE].

[56] M. Czakon and A. Mitov, Top++: a program for the calculation of the top-pair cross-section at hadron colliders, Comput. Phys. Commun. 185 (2014) 2930 [arXiv:1112.5675] [InSPIRE]. 
[57] CMS collaboration, Search for three-jet resonances in pp collisions at $\sqrt{s}=7$ TeV, Phys. Lett. $B 718$ (2012) 329 [arXiv: 1208.2931] [INSPIRE].

[58] ATLAS collaboration, Search for pair production of massive particles decaying into three quarks with the ATLAS detector in $\sqrt{s}=7$ TeV pp collisions at the LHC, JHEP 12 (2012) 086 [arXiv: 1210.4813] [INSPIRE].

[59] ATLAS collaboration, Search for massive supersymmetric particles decaying to many jets using the ATLAS detector in pp collisions at $\sqrt{s}=8$ TeV, Phys. Rev. D 91 (2015) 112016 [Erratum ibid. 93 (2016) 039901] [arXiv: 1502.05686] [INSPIRE].

[60] M. Redi, V. Sanz, M. de Vries and A. Weiler, Strong signatures of right-handed compositeness, JHEP 08 (2013) 008 [arXiv: 1305.3818] [INSPIRE].

[61] R.H. Dalitz, Decay of $\tau$ mesons of known charge, Phys. Rev. 94 (1954) 1046 [InSPIRE].

[62] T. Junk, Confidence level computation for combining searches with small statistics, Nucl. Instrum. Meth. A 434 (1999) 435 [hep-ex/9902006] [INSPIRE].

[63] A.L. Read, Presentation of search results: the CL $L_{s}$ technique, J. Phys. G 28 (2002) 2693 [INSPIRE].

[64] G. Cowan, K. Cranmer, E. Gross and O. Vitells, Asymptotic formulae for likelihood-based tests of new physics, Eur. Phys. J. C 71 (2011) 1554 [Erratum ibid. 73 (2013) 2501] [arXiv:1007.1727] [INSPIRE].

[65] CDF collaboration, First search for multijet resonances in $\sqrt{s}=1.96$ TeV p $\bar{p}$ collisions, Phys. Rev. Lett. 107 (2011) 042001 [arXiv:1105.2815] [INSPIRE].

[66] ALEPH collaboration, Search for R-parity violating decays of supersymmetric particles in $e^{+} e^{-}$collisions at center-of-mass energies near 183 GeV, Eur. Phys. J. C 13 (2000) 29 [INSPIRE].

[67] ALEPH collaboration, Search for supersymmetric particles with $R$ parity violating decays in $e^{+} e^{-}$collisions at $\sqrt{s}$ up to 209 GeV, Eur. Phys. J. C 31 (2003) 1 [hep-ex/0210014] [INSPIRE].

[68] CMS collaboration, Measurement of the $t \bar{t}$ production cross section in the all-jet final state in pp collisions at $\sqrt{s}=7 \mathrm{TeV}$, JHEP 05 (2013) 065 [arXiv:1302.0508] [INSPIRE].

[69] ATLAS collaboration, Measurement of the top-quark mass in the fully hadronic decay channel from ATLAS data at $\sqrt{s}=7$ TeV, Eur. Phys. J. C 75 (2015) 158 [arXiv:1409.0832] [INSPIRE].

[70] CMS collaboration, Measurement of the $t \bar{t}$ production cross section in the all-jets final state in pp collisions at $\sqrt{s}=8$ TeV, Eur. Phys. J. C 76 (2016) 128 [arXiv:1509.06076] [INSPIRE].

[71] ATLAS collaboration, Top-quark mass measurement in the all-hadronic $t \bar{t}$ decay channel at $\sqrt{s}=8 \mathrm{TeV}$ with the ATLAS detector, JHEP 09 (2017) 118 [arXiv:1702.07546] [INSPIRE].

[72] ATLAS collaboration, Measurements of top-quark pair single-and double-differential cross-sections in the all-hadronic channel in pp collisions at $\sqrt{s}=13$ TeV using the ATLAS detector, arXiv:2006.09274 [INSPIRE].

[73] ATLAS collaboration, Search for R-parity-violating supersymmetric particles in multi-jet final states produced in pp collisions at $\sqrt{s}=13 \mathrm{TeV}$ using the ATLAS detector at the LHC, Phys. Lett. B 785 (2018) 136 [arXiv:1804.03568] [INSPIRE]. 
[74] CMS collaboration, Searches for light- and heavy-flavour three-jet resonances in pp collisions at $\sqrt{s}=8 \mathrm{TeV}$, Phys. Lett. B $\mathbf{7 3 0}$ (2014) 193 [arXiv:1311.1799] [InSPIRE].

[75] M. Cacciari, G.P. Salam and G. Soyez, The anti- $k_{t}$ jet clustering algorithm, JHEP 04 (2008) 063 [arXiv: 0802.1189] [INSPIRE].

[76] J. Alwall et al., Comparative study of various algorithms for the merging of parton showers and matrix elements in hadronic collisions, Eur. Phys. J. C 53 (2008) 473 [arXiv:0706.2569] [INSPIRE].

[77] T. Gleisberg et al., Event generation with SHERPA 1.1, JHEP 02 (2009) 007 [arXiv:0811.4622] [INSPIRE]. 